Author affiliations appear at the end of this article.

Submitted May 22, 2011; accepted October 19, 2011; published online ahead of print at www.jco.org on December 19, 2011

Written on behalf of the European Organisation for Research and Treatment of Cancer (EORTC) Lymphoma Group and Groupe d'Étude des Lymphomes de l'Adulte.

Supported by a research grant from the Lance Armstrong Foundation and by a travel grant from the René Vogels

Stichting (M.A.E.v.d.K.)

M.H.-A. and H.C.K.-N. contributed equally to this work.

The contents of this publication and methods used are solely the responsibility of the authors and do not necessarily represent the official views of the EORTC headquarters.

Authors' disclosures of potential conflicts of interest and author contributions are found at the end of this article.

Corresponding author: Prof. Dr. Hanneke C. Kluin-Nelemans, Department of Hematology, University Medical Center Groningen, University of Groningen, DA21, Hanzeplein 1, 9700 RB, Groningen, the Netherlands; e-mail: j.c.kluin@int.umcg.nl.

(c) 2011 by American Society of Clinical Oncology

0732-183X/12/3003-291/\$20.00

DOI: 10.1200/JCO.2011.37.1989

\title{
Premature Ovarian Failure and Fertility in Long-Term Survivors of Hodgkin's Lymphoma: A European Organisation for Research and Treatment of Cancer Lymphoma Group and Groupe d'Étude des Lymphomes de l'Adulte Cohort Study
}

Marleen A.E. van der Kaaij, Natacha Heutte, Paul Meijnders, Edwige Abeilard-Lemoisson, Michele Spina, Elizabeth C. Moser, Anouk Allgeier, Bart Meulemans, Arnold H.M. Simons, Pieternella J. Lugtenburg, Berthe M.P. Aleman, Evert M. Noordijk, Christophe Fermé, José Thomas, Aspasia Stamatoullas, Christophe Fruchart, Pauline Brice, Isabelle Gaillard, Serge Bologna, Francisca Ong, Houchingue Eghbali, Jeanette K. Doorduijn, Franck Morschhauser, Catherine Sebban, Judith M. Roesink, Marie Bouteloup, Achiel Van Hoof, John M.M. Raemaekers, Michel Henry-Amar, and Hanneke C. Kluin-Nelemans

$$
\begin{array}{llllllll}
\text { A } & \text { B } & \text { S } & \text { T } & \text { R } & \text { A } & \text { C } & \text { T }
\end{array}
$$

\section{Purpose}

In this large cohort of Hodgkin's lymphoma survivors with long follow-up, we estimated the impact of treatment regimens on premature ovarian failure (POF) occurrence and motherhood, including safety of nonalkylating chemotherapy and dose-response relationships for alkylating chemotherapy and age at treatment.

\section{Patients and Methods}

The Life Situation Questionnaire was sent to 1,700 women treated in European Organisation for Research and Treatment of Cancer and Groupe d'Étude des Lymphomes de I'Adulte trials between 1964 and 2004. Women treated between ages 15 and 40 years and currently not using hormonal contraceptives ( $n=460$ ) were selected to assess occurrence of POF. Cumulative POF risk was estimated using the life-table method. Predictive factors were assessed by Cox regression analysis.

\section{Results}

Median follow-up was 16 years (range, 5 to 45 years). Cumulative risk of POF after alkylating chemotherapy was $60 \%(95 \% \mathrm{Cl}, 41 \%$ to $79 \%)$ and only $3 \%(95 \% \mathrm{Cl}, 1 \%$ to $7 \%)$ after nonalkylating chemotherapy (doxorubicin, bleomycin, vinblastine, and dacarbazine; epirubicin, bleomycin, vinblastine, and prednisone). Dose relationship between alkylating chemotherapy and POF occurrence was linear. POF risk increased by $23 \%$ per year of age at treatment. In women treated without alkylating chemotherapy at age younger than 32 years and age 32 years or older, cumulative POF risks were $3 \%(95 \% \mathrm{Cl}, 1 \%$ to $16 \%)$ and $9 \%(95 \% \mathrm{Cl}, 4 \%$ to $18 \%)$, respectively. If menstruation returned after treatment, cumulative POF risk was independent of age at treatment. Among women who ultimately developed POF, 22\% had one or more children after treatment, compared with $41 \%$ of women without POF.

\section{Conclusion}

Nonalkylating chemotherapy carries little to no excess risk of POF. Dose-response relationships for alkylating chemotherapy and age at treatment are both linear. Timely family planning is important for women at risk of POF.

\section{J Clin Oncol 30:291-299. (C) 2011 by American Society of Clinical Oncology}

\section{INTRODUCTION}

High cure rates (80\% to $90 \%$ ) of Hodgkin's lymphoma (HL) and a predominant incidence in patients age 20 to 44 years have resulted in many patients wishing to start or complete a family after treatment. ${ }^{1-3}$ Since 1964, the European Organisa- tion for Research and Treatment of Cancer (EORTC) Lymphoma Group (in collaboration with the Groupe d'Etude des Lymphomes de l'Adulte [GELA] since 1993), has treated patients with HL uniformly in clinical trials. From 1982 onward, the aim of these trials has also focused on reduction of treatment-related toxicity. 
Whereas in men fertility is relatively easily studied by analysis of sperm counts and follicle stimulating hormone, ${ }^{4,5}$ hormone measurements in women are influenced by menstrual cycle patterns and hormonal contraceptive use. Pregnancy outcomes and documentation of (premature) cessation of menses offer reliable information. Premature ovarian failure (POF), defined as irreversible amenorrhea lasting more than 12 months before the age of 40 years, can develop during treatment or occur years later, reflecting early depletion of oocytes caused by gonadotoxic treatment. Interaction between the gonadotoxic drug and menstrual cycle in women can be complex as regards time. Women may cease menstruating during therapy, recover thereafter, and become pregnant but still experience POF or early menopause (between ages 40 and 44 years) later on. ${ }^{6}$

Few reports on ovarian failure and infertility after treatment for HL have provided long-term follow-up. ${ }^{7,8}$ Most have involved low patient numbers or women treated in childhood. ${ }^{8-10}$ Information on both menstrual status and number of children is even rarer. In addition, almost no data have been published on nonalkylating chemotherapy, which is presently considered first-choice therapy in earlystage disease. ${ }^{9}$ In 2009, the Life Situation Questionnaire (LSQ) was sent to HL survivors treated within EORTC-GELA trials. This questionnaire addressed in detail fertility and parenthood. It offered the opportunity to study fertility outcome in a large cohort of long-term survivors and compare the influence of various treatment regimens on POF occurrence and age at menopause as well as on female fertility by comparing chances of motherhood after HL.

\section{PATIENTS AND METHODS}

\section{Women}

Overall, 3,020 women were included in nine consecutive randomized clinical trials conducted in 13 European countries between 1964 and 2004 (Table 1). Protocols of the H1 through H9 trials are detailed in published reports. ${ }^{2,11-19}$ Patients presented with histologically proven, previously untreated HL. Exclusion criteria were concomitant or previous malignancies or serious comorbidities. Treatment administered was recorded prospectively, including chemotherapy type, number of cycles, radiotherapy field, and dose. In 2008, after human investigations committee approval, current addresses were tracked for all 2,712 women alive at last follow-up. For 1,700 women from five countries and 114 hospitals, a recent address was obtained (Fig 1).

\section{Questionnaire}

The LSQ addresses issues not yet available in any validated questionnaire. It contains the following items: parenthood after HL; education, work, and insurance; health and social situation; and support received during treatment. Occurrence of menopause was evaluated based on the following question: "At this moment, do you have a menstrual cycle? If no, is this because of a) menopause b) removal of uterus and/or ovaries c) pregnancy or breastfeeding d) continuous contraceptive use or e) other, please specify." Age at last menstruation was also recorded, including whether this last menstruation was more than 1 year ago. Occurrence of immediate menopause during treatment was evaluated based on the following question: "During first treatment for Hodgkin's lymphoma, did you have a spontaneous menstrual cycle? If no, did your menstrual cycle spontaneously return after treatment?" In the LSQ, data on embryo, oocyte, and ovarian cryopreservation were collected. However, none of these options had resulted in any live births among our patient group.

Of 1,700 women approached, 964 (57\%) returned the questionnaire and provided written informed consent. A nonresponder analysis performed to compare those who did not reply with those who did indicated almost no differences. Responders came more often from earlier trials and thus were slightly less often treated with nonalkylating chemotherapy and radiotherapy above the diaphragm. Disease stage distribution was similar in responders and nonresponders.

\section{Selection of Women}

For the POF analysis, we included all women age 15 years or older at treatment who had completed the questions on menstruation and menopause $(\mathrm{n}=945)$. Women who were (post) menopausal before treatment were excluded, as were those with unknown menstrual status because of current contraceptive use. Women who had had sterilizing surgery (removal of uterus and/or ovaries) at an unknown date and women with menopause resulting from an unrelated medical event were also excluded.

Because only effects of primary treatment were considered, women with nonresponse or progression during treatment were excluded; those who experienced a relapse were censored at time of relapse. Rates of progression or relapse were low ( 82 of 964 patients; $8.5 \%$ ). Women with a second malignancy were censored at time of that diagnosis, as were those with surgical menopause at time of surgery and those with continuous progestagen use at start of use, if dates were provided. Analysis was performed on treatment actually received, not on an intention-to-treat basis. Sixteen women received several chemotherapy regimens (protocol violation) and were excluded from analysis. After exclusions, 575 women were suitable for analysis; 460 were younger than 40 years of age at treatment for HL and therefore at risk for POF. They were included in analyses with POF as the end point (Fig 1).

\section{Definitions}

POF was defined as menopause before age 40 years. Menopause was defined as cessation of menstruation at least 1 year before the date of the survey in the absence of pregnancy, breastfeeding, continuous use of progestagens or other medication causing amenorrhea, or surgical removal of uterus and/ or ovaries.

Women administered chemotherapy were grouped into those treated with regimens without alkylating agents (doxorubicin, bleomycin, vinblastine, and dacarbazine $[\mathrm{ABVD}]^{20}$; epirubicin, bleomycin, vinblastine, and prednisone $[\mathrm{EBVP}]^{21}$ ) and those treated with alkylating chemotherapy (mechlorethamine, vincristine, procarbazine, and prednisone $[\mathrm{MOPP}]^{22}$; MOPP/doxorubicin, bleomycin, and vinblastine [MOPP/ABV hybrid $[\mathrm{RSQB}]^{23}$; cyclophosphamide, doxorubicin, vincristine, bleomycin, etoposide, procarbazine, and prednisone [standard BEACOPP $\left.{ }^{24}\right)$. Of all women included, $11 \%$ to $12 \%$ received ABVD, $18 \%$ to $19 \%$ received EBVP, $6 \%$ received $\mathrm{MOPP}, 33 \%$ to $36 \%$ received $\mathrm{MOPP} / \mathrm{ABV}$, and $4 \%$ received standard BEACOPP. None received dose-escalated BEACOPP (Appendix Table A1, online only). Cycles administered at reduced doses were considered full cycles. The number of cycles was used to estimate the amount of alkylating chemotherapy administered, counting one cycle of MOPP/ABV or BEACOPP as equivalent to 0.5 MOPP cycle. In two trials, maintenance chemotherapy for 2 years was administered: vinblastine 10 or $6 \mathrm{mg}$, or $6 \mathrm{mg}$ weekly for 3 months alternating with procarbazine daily $150 \mathrm{mg}$ for 3 weeks, with a gap of at least 4 weeks in between. ${ }^{11,12}$ Because the cumulative procarbazine dose in this regimen was $9 \mathrm{~g} / \mathrm{m}^{2}$, approximately comparable to six MOPP cycles, procarbazine-containing maintenance therapy was considered equivalent to six cycles of MOPP.

Information on smoking, education level, and number of biologic and adopted children was obtained from the LSQ questionnaire. Education level was grouped into low, middle, and high: low comprised primary school and the lower level of secondary school (corresponding to International Standard Classification of Education [ISCED] levels ${ }^{25} 0,1$, and 2); middle, the higher levels of secondary school (ISCED levels 3 and 4); and high, higher education and university (ISCED levels 5 and 6).

\section{Statistical Analysis}

The aim of this study was to estimate the impact of HL treatment regimens on POF occurrence (primary end point), menopause, and motherhood. A cohort analysis was performed. Women were observed from start of HL treatment until POF $(\mathrm{n}=77)$, age 40 years $(\mathrm{n}=202)$, relapse $(\mathrm{n}=39)$, second malignancy $(n=8)$, surgical menopause $(n=4)$, first continuous progestagen use $(n=10)$, or date of LSQ completion if younger than 40 years and still menstruating $(\mathrm{n}=120)$. 


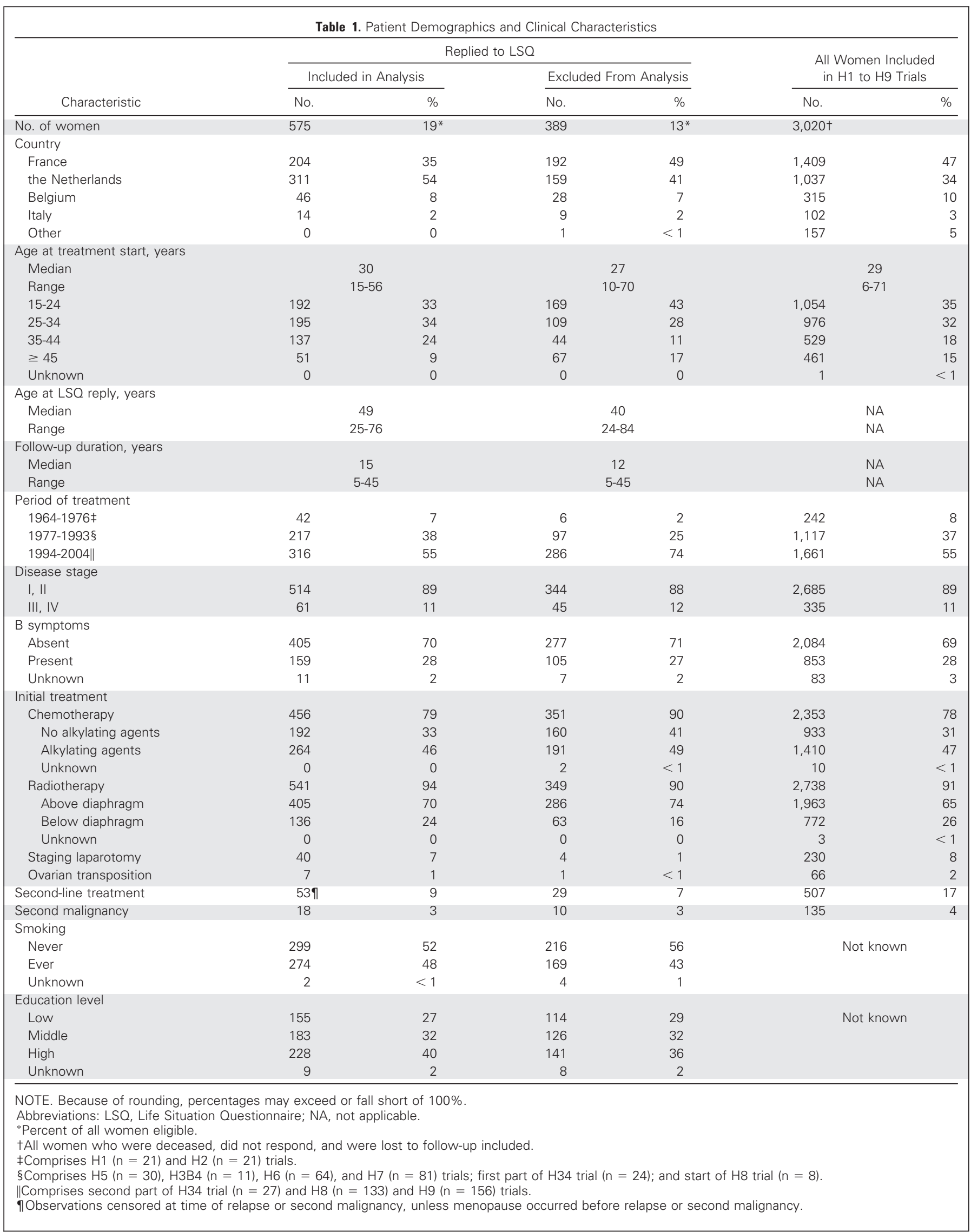




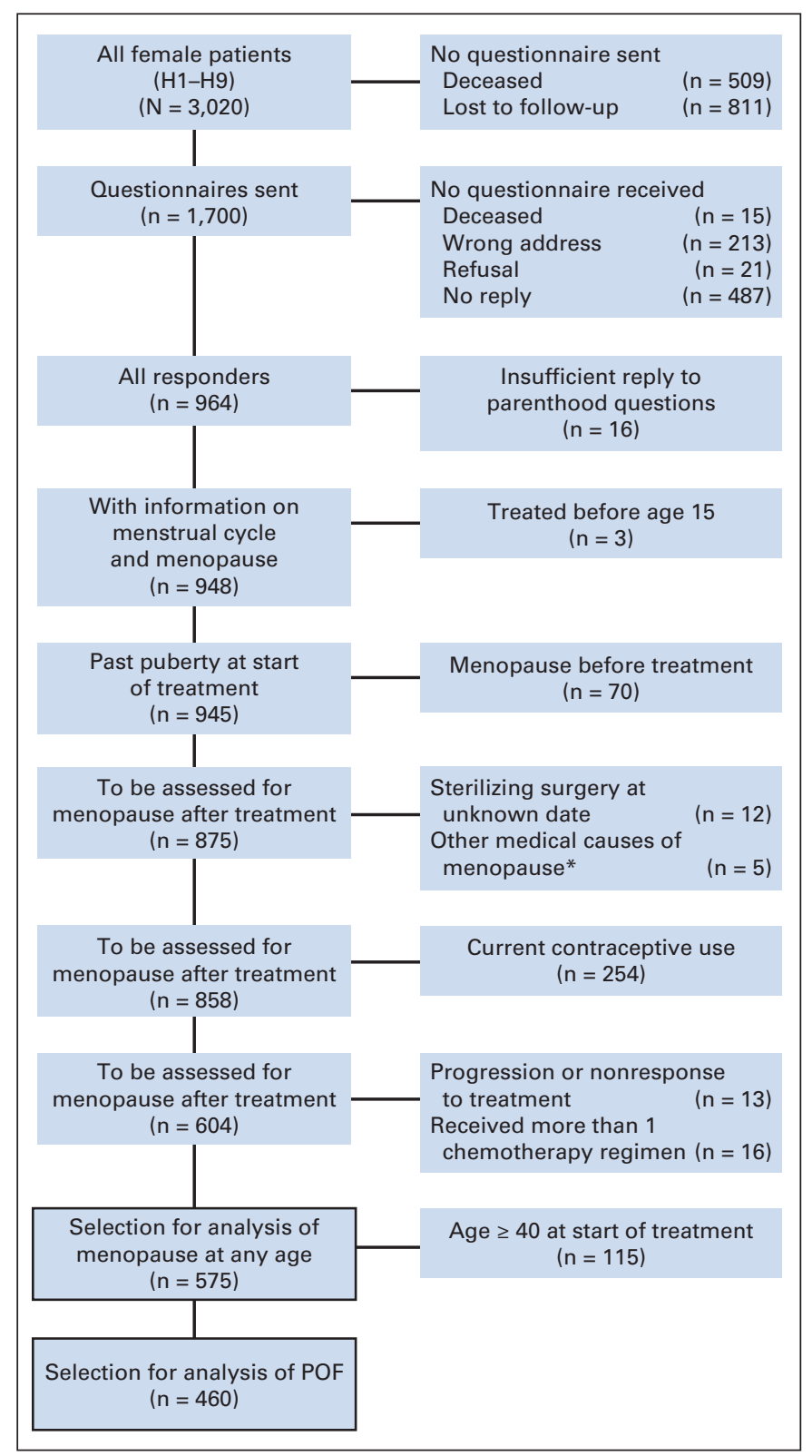

Fig 1. CONSORT diagram; selection of women included in analysis. ${ }^{*}$ ) Menopause after thyroid problems, curettage (Asherman's syndrome), syndrome of Mayer-Rokitansky-Küster, cerebrovascular accident, and endometriosis (one patient case in each category).

Time to POF was defined as time from start of treatment to POF occurrence, to age 40 years, or to LSQ reply, whichever came first. Cumulative risks of POF and exact binomial 95\% CIs were estimated using the life-table method. ${ }^{26}$ Univariable analysis of factors influencing POF occurrence and menopause age was performed using the Kaplan-Meier method and log-rank test. A multivariable analysis of prognostic factors with regard to POF occurrence was performed using Cox regression analysis. Variables entered into the model were treatment age; chemotherapy (alkylating, nonalkylating) or cycles of alkylating chemotherapy in MOPP equivalents (no alkylating chemotherapy, $<$ four cycles of MOPP equivalent, $\geq$ four cycles of MOPP equivalent); radiotherapy (above or below diaphragm or on iliac region); second-line treatment (no, yes); treatment period (1964 to 1976, 1977 to 1993, 1994 to 2004); clinical stage (I to II, III to IV); presence of B symptoms (no, yes); smoking (never, ever); and education level (low, middle, high). A stepwise selection procedure was used. The likelihood ratio test was used to compare a model with age or cycles of MOPP equivalent as continuous variables against models with age or cycles of MOPP equivalent as dichotomous, categorical, logarithmic, or continuous quadratic variables (results shown in part). By receiver operating characteristic analysis, the optimal cutoff for treatment age was evaluated in predicting POF occurrence. Exact binomial 95\% CIs for sensitivity and specificity were calculated. All statistical tests were two sided, and statistical significance was defined as a $P$ value of less than .01 to adjust for multiple comparisons.

\section{RESULTS}

Characteristics for all women included in the trials $(\mathrm{N}=3,020)$ and those selected for analysis are listed in Table $1(\mathrm{n}=575)$ and Table 2 (selection POF, $\mathrm{n}=460$ ). Women selected were more often treated 10 or more years ago, because the menstrual status of women using hormonal contraceptives (on average younger) could not be evaluated. Women selected for analysis of POF were on average younger at treatment because those age 40 years or older were no longer at risk for POF. Clinical stage distribution was similar in the groups.

After median follow-up of 16 years (range, 5 to 45 years), 77 of 460 women developed POF. To avoid overestimation of POF incidence compared with general population data, we hypothesized all women excluded from analysis because of use of hormonal contraceptives still had a menstrual cycle. Being younger, these women were more probably fertile than those who did not use hormonal contraceptives. Under this most conservative approach possible, the corrected incidence of POF in our cohort was 20.1\% (95\% CI, $15.9 \%$ to $24.8 \%$ ) compared with $0.9 \%(95 \% \mathrm{CI}, 0.3 \%$ to $1.5 \%)$ in the general population. ${ }^{27}$

Twenty-three percent of women were treated with radiotherapy alone (of whom one received iliac radiotherapy), 33\% with combined chemoradiotherapy without alkylating agents and 44\% with combined radiotherapy and alkylating agents. The distribution of treatment and other characteristics according to POF occurrence is shown in Table 2. Sixty-nine (34\%) of 202 women treated with alkylating chemotherapy developed POF, whereas among women treated with nonalkylating chemotherapy, four (3\%) of $151 \mathrm{did}$. Among women treated with iliac-region radiotherapy, 11 (41\%) of 27 had POF, but all had also been treated with alkylating chemotherapy.

\section{Multivariable Analysis}

Figures $2 \mathrm{~A}$ to $2 \mathrm{D}$ illustrate treatment (alkylating chemotherapy, yes or no) and treatment age influence on age of menopause. Women treated with alkylating chemotherapy had an age-adjusted hazard ratio (HR) of 12.31 (95\% CI, 5.90 to $25.68 ; P<.001)$ for POF occurrence compared with women not treated with alkylating chemotherapy (Table 2). The cumulative risk of POF for treatment with alkylating chemotherapy $(\mathrm{n}=202)$ was $60 \%(95 \% \mathrm{CI}, 41 \%$ to $79 \%$ ) and only $3 \%(95 \% \mathrm{CI}, 1 \%$ to $7 \%$ ) or $6 \%(95 \% \mathrm{CI}, 2 \%$ to $20 \%$ ) for treatment with nonalkylating chemotherapy (ABVD or EBVP, $\mathrm{n}=151)$ or radiotherapy only $(\mathrm{n}=107)$, respectively. The adjusted HRs were 9.84 (95\% CI, 4.62 to $20.97 ; P<.001$ ) for women treated with fewer than four cycles of MOPP-equivalent chemotherapy and 21.10 (95\% CI, 9.49 to $46.93 ; P<.001)$ for 


\begin{tabular}{|c|c|c|c|c|c|c|c|c|c|c|c|c|c|}
\hline \multirow[b]{2}{*}{ Characteristic } & \multicolumn{2}{|c|}{ Total } & \multicolumn{2}{|c|}{ POF } & \multicolumn{2}{|c|}{ No POF } & \multirow[b]{2}{*}{$P^{*}$} & \multicolumn{3}{|c|}{ Model With All Variables } & \multicolumn{3}{|c|}{ Final Model } \\
\hline & No. & $\%$ & No. & $\%$ & No. & $\%$ & & $H R$ & $95 \% \mathrm{Cl}$ & $P$ & $H R$ & $95 \% \mathrm{Cl}$ & $P$ \\
\hline No. of women & 460 & & 77 & $17 \dagger$ & 383 & $83+$ & & & & & & & \\
\hline Age at start of treatment, years & & & & & & & $<.001$ & $1.23 \neq$ & 1.16 to 1.30 & $<.001$ & $1.23 \neq$ & 1.16 to 1.30 & $<.001$ \\
\hline $15-24$ & 192 & 42 & 14 & 18 & 178 & 46 & & & & & & & \\
\hline $25-34$ & 195 & 42 & 44 & 57 & 151 & 39 & & & & & & & \\
\hline $35-39$ & 73 & 16 & 19 & 25 & 54 & 14 & & & & & & & \\
\hline Treatment period & & & & & & & .004 & & & & & & \\
\hline $1964-1976$ & 42 & 9 & 1 & 1 & 41 & 11 & & 1.00 & & & - & & \\
\hline 1977-1993 & 188 & 41 & 43 & 56 & 145 & 38 & & 1.80 & 0.22 to 15.05 & .59 & & & \\
\hline $1994-2004$ & 230 & 50 & 33 & 43 & 197 & 51 & & 1.52 & 0.18 to 13.02 & .70 & & & \\
\hline Disease stage & & & & & & & $<.001$ & & & & & & \\
\hline I, II & 409 & 89 & 54 & 70 & 355 & 93 & & 1.00 & & & - & & \\
\hline III, IV & 51 & 11 & 23 & 30 & 28 & 7 & & 1.32 & 0.68 to 2.56 & .41 & & & \\
\hline B symptoms & & & & & & & $<.001$ & & & & & & \\
\hline Absent & 323 & 70 & 36 & 47 & 287 & 75 & & 1.00 & & & - & & \\
\hline Present & 130 & 28 & 40 & 52 & 90 & 24 & & 1.32 & 0.80 to 2.17 & .27 & & & \\
\hline Unknown & 7 & 1 & 1 & 1 & 6 & 2 & & & & & & & \\
\hline \multicolumn{14}{|l|}{ Initial treatment } \\
\hline Chemotherapy & 353 & 77 & 73 & 95 & 280 & 73 & $<.001$ & & & & & & \\
\hline No alkylating agents & 151 & 33 & 4 & 5 & 147 & 38 & & 1.00 & & & 1.00 & & \\
\hline Alkylating agents§ & 202 & 44 & 69 & 90 & 133 & 35 & $<.001$ & & & & 12.31 & 5.90 to 25.68 & $<.001$ \\
\hline $\begin{array}{l}1.5 \text { to three cycles of MOPP } \\
\text { equivalent }\end{array}$ & 155 & 34 & 44 & 57 & 111 & 29 & & 8.49 & 3.75 to 19.23 & $<.001$ & 9.84 & 4.62 to 20.97 & $<.001$ \\
\hline $\begin{array}{l}\geq \text { Four cycles of MOPP } \\
\text { equivalent }\end{array}$ & 47 & 10 & 25 & 29 & 22 & 6 & & 15.04 & 6.14 to 36.87 & $<.001$ & 21.10 & 9.49 to 46.93 & $<.001$ \\
\hline Radiotherapy\| & 429 & 93 & 69 & 90 & 360 & 94 & $<.001$ & & & & & & \\
\hline Above diaphragm & 319 & 69 & 53 & 69 & 266 & 69 & & 1.00 & & & & & \\
\hline Below diaphragm, not iliac region & 83 & 18 & 5 & 6 & 78 & 20 & & 0.83 & 0.32 to 2.16 & .70 & & & \\
\hline Iliac region & 27 & 6 & 11 & 14 & 16 & 4 & $<.001$ & 0.76 & 0.35 to 1.66 & .49 & & & \\
\hline Smoking & & & & & & & .186 & & & & & & \\
\hline Never & 237 & 52 & 35 & 45 & 202 & 53 & & 1.00 & & & - & & \\
\hline Ever & 222 & 48 & 42 & 55 & 180 & 47 & & 0.86 & 0.54 to 1.38 & .54 & & & \\
\hline Unknown & 1 & $<1$ & 0 & 0 & 1 & $<1$ & & & & & & & \\
\hline \multicolumn{14}{|c|}{$\begin{array}{l}\text { NOTE. Because of rounding, percentages may exceed or fall short of } 100 \% \text {. } \\
\text { Abbreviations: HR, hazard ratio; MOPP, mechlorethamine, vincristine, procarbazine, and prednisone; POF, premature ovarian failure. } \\
\text { *Log-rank test. } \\
\text { †Percent of all women included. } \\
\text { łAge analyzed as continuous variable. } \\
\text { §Either alkylating agents as dichotomous (yes or no) or categorical variable (no alkylating agents, } 1.5 \text { to three cycles of MOPP equivalent, } \geq \text { four cycles of MOPP } \\
\text { equivalent) were entered into multivariable model; difference between chemotherapy without alkylating agents and radiotherapy only was not statistically significant. } \\
\text { \|Radiotherapy on iliac region (no or yes) and combination variable of radiotherapy and alkylating chemotherapy (no alkylating chemotherapy and no radiotherapy } \\
\text { below diaphragm, radiotherapy below diaphragm but no alkylating chemotherapy, alkylating chemotherapy but no radiotherapy below diaphragm, or both) were not } \\
\text { statistically significant. }\end{array}$} \\
\hline
\end{tabular}

women treated with four or more cycles compared with those treated without alkylating chemotherapy (Table 2). Number of cycles of MOPP equivalent (categorical) was highly significant $(P<.001)$ when entered into the Cox regression model for POF risk, which included only women treated with alkylating chemotherapy, indicating a significant dose-response relationship (Fig 3). Comparing the model with cycles of MOPP equivalent as continuous variable against the model with cycles of MOPP of equivalent as quadratic or logarithmic variable showed no statistically significant difference $(P=.92$ and $P=.30$, respectively), indicating a linear dose-response relationship. The HR per cycle of alkylating chemotherapy was 1.50 (95\% CI, 1.37 to $1.64 ; P<.001)$.

There was a $23 \%$ increase in POF risk per year of age at treatment (Table 2). Comparing the model with treatment age as continuous variable against the model with treatment age as quadratic or logarithmic variable showed no statistically significant difference $(P=.16$ and
$P=.19$, respectively), indicating a linear relationship. However, when actual calendar age attained at end of follow-up was used as a time variable in survival analysis, the difference between age groups at treatment was much smaller. The total cumulative risks of POF were $17 \%$ (95\% CI, 9\% to 30\%) for those younger than age 25 years at treatment and 32\% (95\% CI, 20\% to 47\%) for those 35 years of age or older, respectively, but the difference was mostly the result of direct menopause during treatment $(n=94)$. If only women with recurring menstruation after treatment were included, the cumulative risks of POF were $17 \%$ (95\% CI, $9 \%$ to $30 \%$ ) and $16 \%$ (95\% CI, $7 \%$ to $35 \%$ ), respectively. This illustrates that with long-term follow-up, women without direct acute ovarian failure at time of treatment had a comparative cumulative risk of developing POF, regardless of treatment age.

We further evaluated prognostic value of treatment age for POF occurrence by calculating receiver operating characteristic curves for 


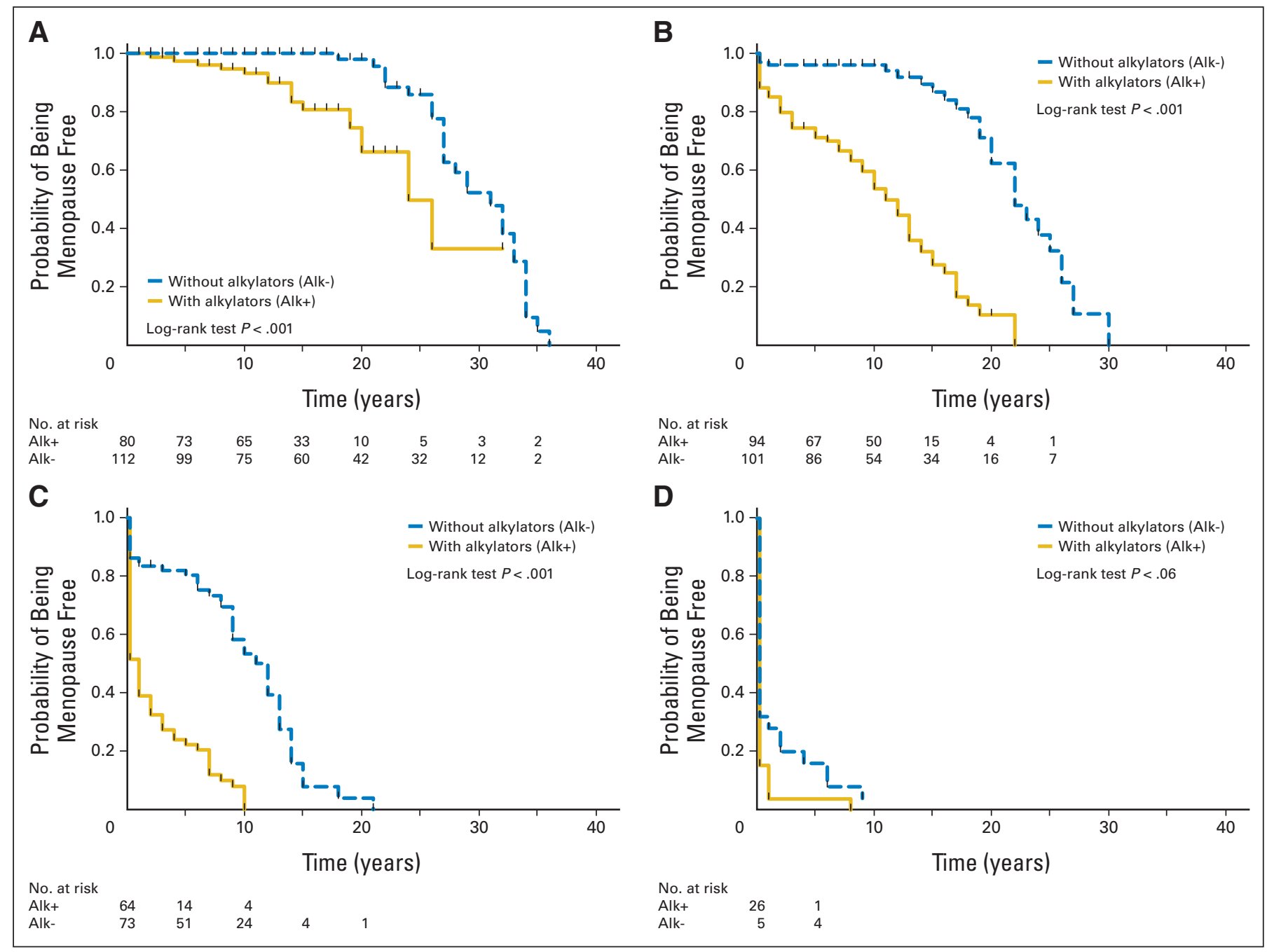

Fig 2. Time to menopause after treatment for Hodgkin's lymphoma by age at start of treatment: (A) 15 to 24 , (B) 25 to 34 , (C) 35 to 44 , and (D) 45 years or older. Alk+, chemotherapy containing alkylating agents with or without radiotherapy $(n=264)$; Alk - , chemotherapy not containing alkylating agents with or without radiotherapy or radiotherapy alone $(n=311)$.

several cutoff values. The effect of age on POF occurrence was smaller in women treated with alkylating chemotherapy (area under the curve, 0.75 ; $95 \%$ CI, 0.54 to 0.96 ) compared with women treated without alkylating chemotherapy (area under the curve, $0.68 ; 95 \% \mathrm{CI}$, 0.61 to 0.76 ; Figs $4 \mathrm{~A}, 4 \mathrm{~B})$. In the latter, the best cutoff value was treatment before age 32 years versus treatment at a later age. Sensitivity was $88 \%$ (95\% CI, $83 \%$ to $91 \%)$ and specificity was $71 \%$ (95\% CI, $65 \%$ to $77 \%$ ). For a cutoff at age 30 years (cutoff usually selected in literature), specificity was $63 \%$ (95\% CI, 57\% to 69\%). We found no statistically significant interaction between treatment age as continuous $(P=.18)$ or categorical variable $(P=.61)$ and the number of cycles of MOPP equivalent as categorical variable. The cumulative risks for POF in women treated at age younger than 32 years and age 32 years or older without alkylating chemotherapy were $3 \%$ (95\% CI, $1 \%$ to $16 \%$ ) and $9 \%$ (95\% CI, $4 \%$ to $18 \%$ ), respectively. The HR for POF associated with age 32 years or older at treatment was $15.65(95 \% \mathrm{CI}$, 1.88 to $130 ; P=.01$ ) compared with age younger than 32 years in women treated without alkylating chemotherapy. Other possible risk factors for POF were also assessed. Smoking, B symptoms, clinical stage, and period of treatment had no significant influence on POF occurrence (Table 2).

Overall, 94 women developed acute menopause during first-line treatment: none, $14(15 \%), 41(44 \%)$, and $39(41 \%)$ when ages 15 to 24,25 to 34,35 to 44 , and 45 years or older, respectively. Sixty-four women $(68 \%)$ were treated with alkylating chemotherapy. Of 77 women who developed POF, 17 (22\%) had at least one biologic child after treatment; nine were treated before age 25 years, and eight were from 25 to 32 years of age. This must be compared with 124 (41\%) of 300 of women without POF who had at least one biologic child after treatment.

\section{DISCUSSION}

To our knowledge, this is the first large study to evaluate POF occurrence based on data provided by women themselves, with treatment administered in prospective clinical trials and without masking through contraceptive use. The results highlight several major facts. 


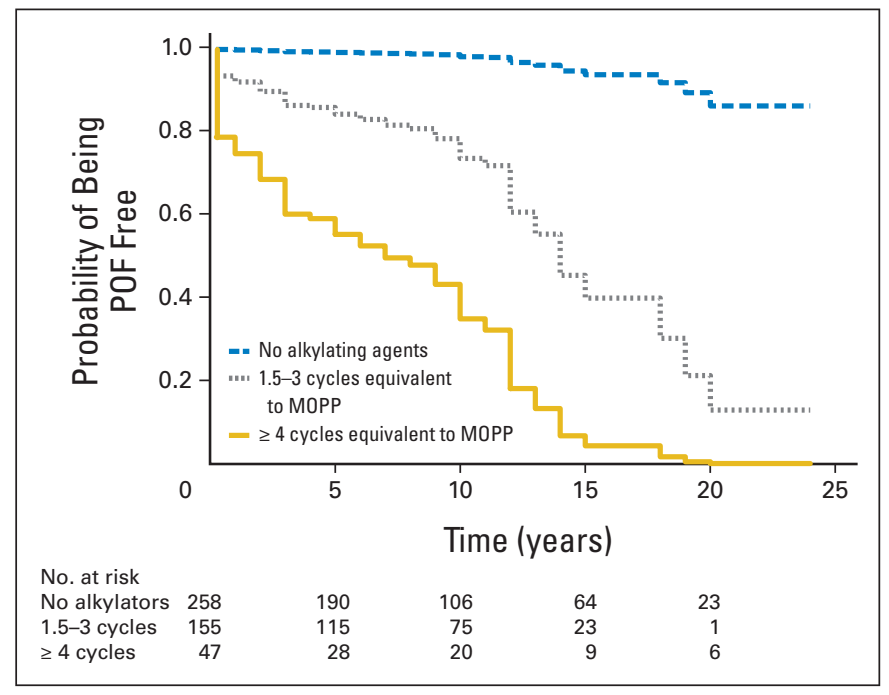

Fig 3. Cox regression estimation of probability of patients free of premature ovarian failure (POF) by time interval since chemotherapy (censored at age 40 years) in 460 women treated for Hodgkin's lymphoma by dose of alkylating chemotherapy, adjusted for age at treatment. MOPP, mechlorethamine, vincristine, procarbazine, and prednisone.

First, we showed nonalkylating chemotherapy carries little excess risk of POF. However, even in this treatment group, chemotherapy administered in women age 32 years or older resulted in an elevated risk of POF development ( $9 \% v 0.9 \%$ in general population). Second, POF occurrence is mainly influenced by alkylating chemotherapy use; the dose relationship between alkylating chemotherapy and POF occurrence is linear. Finally, we can offer insight into the influence of treatment-related POF on motherhood.

The emerging use of (dose-escalated) BEACOPP seems to represent a step backward compared with ABVD from the fertility viewpoint. Behringer et $\mathrm{al}^{28}$ found levels of acute menopause during treatment of $32 \%$ after BEACOPP baseline, 55\% after COPP/ABVD, and even $67 \%$ after escalated BEACOPP. Escalated BEACOPP contains almost double the cyclophosphamide dose and the same procarbazine dose as BEACOPP, so two cycles of escalated BEACOPP can be roughly equated to 1.5 cycles of MOPP, resulting in an HR for POF of 9.84 .

We confirmed the dose-response relationship for procarbazine found by De Bruin et $\mathrm{al}^{7}$ in a study based on medical records not excluding oral contraceptive use. They found an HR of 12.3 for POF after chemotherapy (any type), whereas no significant effect was observed for nonalkylating chemotherapy, used in 50 of 331 women only. The rare reports documenting the effect of nonalkylating chemotherapy on fertility concern limited numbers (24 to 70 women) and sparse data. ${ }^{9,29-31}$

Our data show an increase in risk of $\mathrm{POF}$ of $23 \%$ per year of age at treatment $(\mathrm{HR}, 1.23)$, corrected for dose of alkylating chemotherapy administered. The prognostic value of treatment age for POF occurrence is most pronounced in women receiving nonalkylating chemotherapy. The cutoff age found ( 32 years) is close to that generally used (30 years).

A disadvantage, but simultaneously a strength, of our analysis concerns the information on women currently using hormonal contraception, who had to be excluded because ongoing menstruation

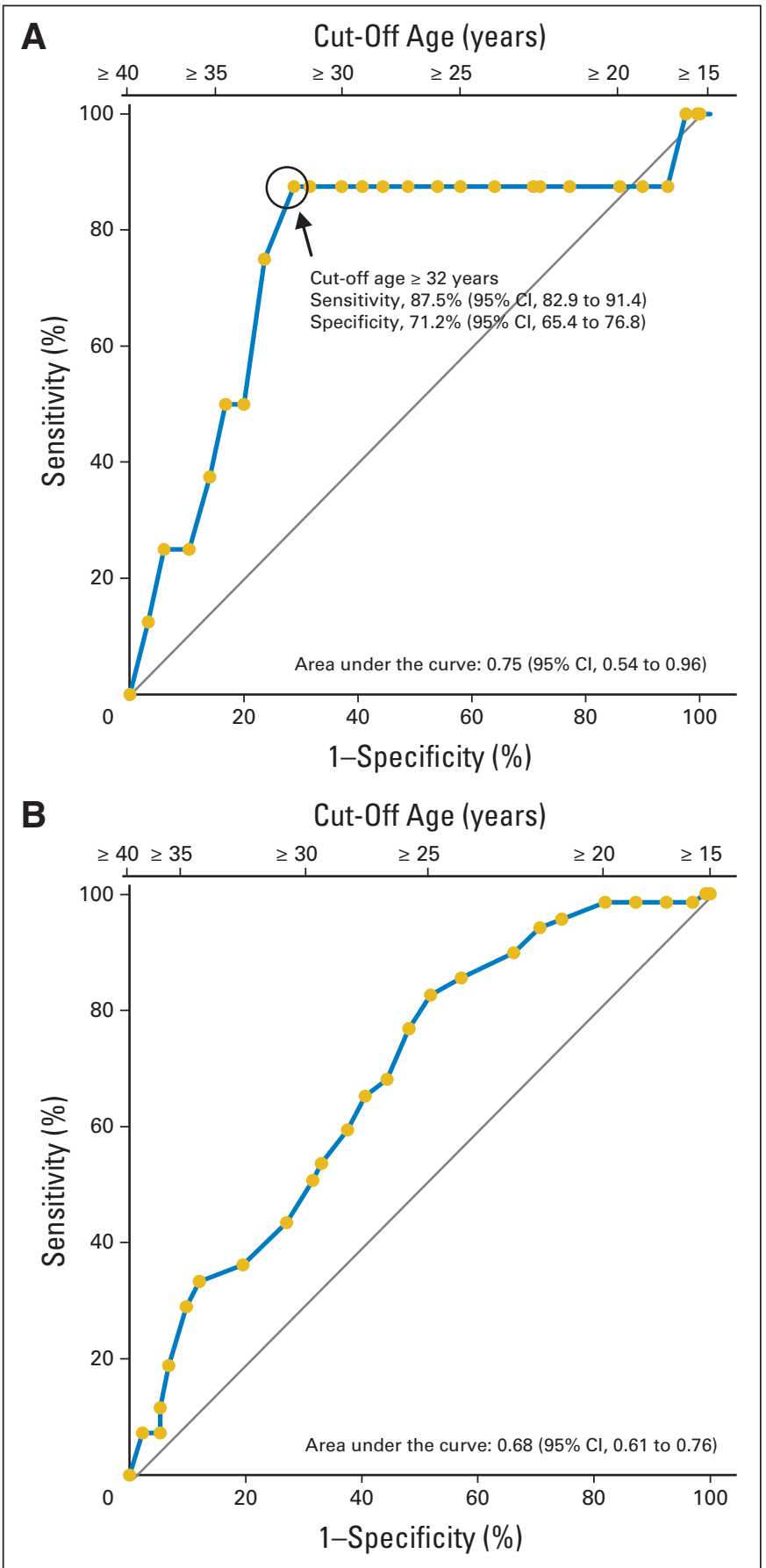

Fig 4. Receiver operating characteristic curves for premature ovarian failure occurrence by treatment age for women treated (A) without and (B) with alkylating chemotherapy.

could mask menopause. A consequence of excluding these on average younger and probably more fertile women is that POF incidences might be inflated. In the comparison with POF incidence in the general population, this has been corrected by using the most conservative estimate possible, assuming that all hormonal contraception users are still menstruating.

Our results are based on a questionnaire filled in by women treated under clinical trial conditions. Correct and current information on menstrual status is best obtained from women themselves, not 
from medical records. However, every questionnaire-based study suffers from response bias. In our study, responders differed slightly from nonresponders in the treatment received but not in treatment age or disease stage. No information on education level was available for nonresponders.

In any survival analysis, the question of censoring is important. In our study, limited numbers were censored because of relapse, second malignancy, or other reasons, so censoring probably did not influence the study results.

Our report is among the few to offer information on the impact of POF on motherhood. Despite treatment toxicity experienced, fortunately, one fourth of women delivered one or more children before POF occurrence. Conversely, our report also indicates that women with proven fertility after treatment can still be confronted with infertility problems at a later stage.

In summary, women treated with nonalkylating chemotherapy younger than 32 years of age have little to no excess POF risk. Alkylating chemotherapy carries a serious POF risk, with HRs of 10 to 21, depending on dose administered. Women with recurring menstruation after treatment should be informed about their potentially shortened fertility lifespan.

\section{AUTHORS' DISCLOSURES OF POTENTIAL CONFLICTS} OF INTEREST

The author(s) indicated no potential conflicts of interest.

\section{AUTHOR CONTRIBUTIONS}

Conception and design: Marleen A.E. van der Kaaij, Elizabeth C. Moser, John M.M. Raemaekers, Michel Henry-Amar, Hanneke C.

Kluin-Nelemans

Financial support: Hanneke C. Kluin-Nelemans

Administrative support: Paul Meijnders, Anouk Allgeier, Bart

Meulemans, Michel Henry-Amar, Hanneke C. Kluin-Nelemans

Provision of study materials or patients: Paul Meijnders, Michele Spina, Pieternella J. Lugtenburg, Berthe M.P. Aleman, Evert M. Noordijk, Christophe Fermé, José Thomas, Aspasia Stamatoullas, Christophe Fruchart, Pauline Brice, Isabelle Gaillard, Serge Bologna, Francisca Ong, Houchingue Eghbali, Jeanette K. Doorduijn, Franck Morschhauser, Catherine Sebban, Judith M. Roesink, Marie Bouteloup, Achiel Van Hoof, John M.M. Raemaekers, Michel Henry-Amar, Hanneke C.

Kluin-Nelemans

Collection and assembly of data: Marleen A.E. van der Kaaij, Natacha Heutte, Paul Meijnders, Edwige Abeilard-Lemoisson, Michele Spina, Anouk Allgeier, Bart Meulemans, Pieternella J. Lugtenburg, Berthe M.P. Aleman, Evert M. Noordijk, Christophe Fermé, José Thomas, Aspasia Stamatoullas, Christophe Fruchart, Pauline Brice, Isabelle Gaillard, Serge Bologna, Francisca Ong, Houchingue Eghbali, Jeanette K. Doorduijn, Franck Morschhauser, Catherine Sebban, Judith M. Roesink, Marie Bouteloup, Achiel Van Hoof, John M.M. Raemaekers, Michel Henry-Amar, Hanneke C. Kluin-Nelemans

Data analysis and interpretation: Marleen A.E. van der Kaaij, Natacha Heutte, Arnold H.M. Simons, Berthe M.P. Aleman, John M.M. Raemaekers, Michel Henry-Amar, Hanneke C. Kluin-Nelemans Manuscript writing: All authors Final approval of manuscript: All authors

\section{REFERENCES}

1. Sieber $M$, Rueffer $U$, Josting $A$, et al: Treatment of Hodgkin's disease: Current strategies of the German Hodgkin's Lymphoma Study Group. Ann Oncol 10:23-29, 1999 (suppl 6)

2. Raemaekers J, Kluin-Nelemans $H$, Teodorovic I, et al: The achievements of the EORTC Lymphoma Group: European Organisation for Research and Treatment of Cancer. Eur J Cancer 38:S107-S113, 2002 (suppl 4)

3. Chen $Y T$, Zheng $\mathrm{T}$, Chou MC, et al: The increase of Hodgkin's disease incidence among young adults: Experience in Connecticut, 19351992. Cancer 79:2209-2218, 1997

4. van der Kaaij MA, Heutte N, Le Stang N, et al: Gonadal function in males after chemotherapy for early-stage Hodgkin's lymphoma treated in four subsequent trials by the European Organisation for Research and Treatment of Cancer: EORTC Lymphoma Group and the Groupe d'Etude des Lymphomes de I'Adulte. J Clin Oncol 25:2825-2832, 2007

5. van der Kaaij MA, Heutte N, van EchtenArends J, et al: Sperm quality before treatment in patients with early stage Hodgkin lymphoma enrolled in EORTC-GELA Lymphoma Group trials. Haematologica 94:1691-1697, 2009

6. van der Kaaij MA, van Echten-Arends J, Simons $A H$, et al: Fertility preservation after chemotherapy for Hodgkin lymphoma. Hematol Oncol 28: 168-179, 2010

7. De Bruin ML, Huisbrink J, Hauptmann M, et al: Treatment-related risk factors for premature menopause following Hodgkin's lymphoma. Blood 111:101-108, 2008
8. Haukvik UK, Dieset I, Bjøro $T$, et al: Treatment-related premature ovarian failure as a long-term complication after Hodgkin's Iymphoma. Ann Oncol 17:1428-1433, 2006

9. Hodgson DC, Pintilie M, Gitterman L, et al: Fertility among female hodgkin lymphoma survivors attempting pregnancy following ABVD chemotherapy. Hematol Oncol 25:11-15, 2007

10. Green DM, Sklar CA, Boice JD Jr, et al: Ovarian failure and reproductive outcomes after childhood cancer treatment: Results from the Childhood Cancer Survivor Study. J Clin Oncol 27:23742381, 2009

11. Tubiana $M$, Henry-Amar $M$, Hayat $M$, et al: Long-term results of the E.O.R.T.C. randomized study of irradiation and vinblastine in clinical stages I and II of Hodgkin's disease. Eur J Cancer 15:645657, 1979

12. Tubiana $M$, Hayat $M$, Henry-Amar $M$, et al: Five-year results of the E.O.R.T.C. randomized study of splenectomy and spleen irradiation in clinical stages I and II of Hodgkin's disease. Eur J Cancer 17:355-363, 1981

13. Carde $P$, Burgers JM, Henry-Amar M, et al: Clinical stages I and II Hodgkin's disease: A specifically tailored therapy according to prognostic factors. J Clin Oncol 6:239-252, 1988

14. Somers $R$, Carde $P$, Henry-Amar $M$, et al: A randomized study in stage IIIB and IV Hodgkin's disease comparing eight courses of MOPP versus an alteration of MOPP with ABVD: A European Organization for Research and Treatment of Cancer Lymphoma Cooperative Group and Groupe Pierreet-Marie-Curie controlled clinical trial. J Clin Oncol 12:279-287, 1994

15. Carde P, Hagenbeek A, Hayat M, et al: Clinical staging versus laparotomy and combined modality with MOPP versus ABVD in early-stage Hodgkin's disease: The H6 twin randomized trials from the European Organization for Research and Treatment of Cancer Lymphoma Cooperative Group. J Clin Oncol 11:2258-2272, 1993

16. Noordijk EM, Carde $P$, Dupouy $N$, et al: Combined-modality therapy for all patients with clinical stage | or II Hodgkin's lymphoma: Long-term results of the European Organisation for Research and Treatment of Cancer $\mathrm{H} 7$ randomized controlled trials. J Clin Oncol 24:3128-3135, 2006

17. Aleman BM, Raemaekers JM, Tirelli U, et al: Involved-field radiotherapy for advanced Hodgkin's lymphoma. N Engl J Med 348:2396-2406, 2003

18. Fermé C, Eghbali $\mathrm{H}$, Meerwaldt JH, et al: Chemotherapy plus involved-field radiation in earlystage Hodgkin's disease. N Engl J Med 357:19161927, 2007

19. Thomas J, Fermé C, Noordijk E, et al: Results of the EORTC-GELA H9 randomized trials: The H9-F trial (comparing 3 radiation dose levels) and $\mathrm{H} 9-\mathrm{U}$ trial (comparing 3 chemotherapy schemes) in patients with favorable or unfavorable early stage Hodgkin's lymphoma (HL). Haematologica 92:27, 2007 (suppl 5; abstr C010)

20. Bonadonna G, Zucali R, Monfardini S, et al: Combination chemotherapy of Hodgkin's disease with adriamycin, bleomycin, vinblastine, and imidazole carboxamide versus MOPP. Cancer 36:252259, 1975

21. Zittoun R, Eghbali $H$, Audebert $A$, et al: The combination of epirubicin, bleomycin, vinblastine and prednisone (EBVP) before radiotherapy in localized stages of Hodgkin's disease: Phase II trials [in French]. Bull Cancer 74:151-157, 1987

22. DeVita VT Jr, Serpick AA, Carbone PP: Combination chemotherapy in the treatment of advanced Hodgkin's disease. Ann Intern Med 73:881-895, 1970 
23. Klimo $P$, Connors JM: MOPP/ABV hybrid program: Combination chemotherapy based on early introduction of seven effective drugs for advanced Hodgkin's disease. J Clin Oncol 3:11741182,1985

24. Diehl $V$, Sieber $M$, Ruffer $U$, et al: BEACOPP: An intensified chemotherapy regimen in advanced Hodgkin's disease-The German Hodgkin's Lymphoma Study Group. Ann Oncol 8:143-148, 1997

25. Organisation for Economic Co-operation and Development: Classifying Educational Programmes: Manual for ISCED-97 Implementation in OECD Countries. Paris, France, Organisation for Economic Co-operation and Development, 1999
26. Schouten LJ, Straatman $H$, Kiemeney LA, et al: Cancer incidence: Life table risk versus cumulative risk. J Epidemiol Community Health 48:596600, 1994

27. Coulam CB, Adamson SC, Annegers JF: Incidence of premature ovarian failure. Obstet Gynecol 67:604-606, 1986

28. Behringer $K$, Breuer $K$, Reineke $T$, et al: Secondary amenorrhea after Hodgkin's lymphoma is influenced by age at treatment, stage of disease, chemotherapy regimen, and the use of oral contraceptives during therapy: A report from the German Hodgkin's Lymphoma Study Group. J Clin Oncol 23:7555-7564, 2005
29. Bonadonna $G$, Bonfante $V$, Viviani $S$, et al: ABVD plus subtotal nodal versus involved-field radiotherapy in early-stage Hodgkin's disease: Longterm results. J Clin Oncol 22:2835-2841, 2004

30. Brusamolino $E$, Lunghi $F$, Orlandi $E$, et al: Treatment of early-stage Hodgkin's disease with four cycles of ABVD followed by adjuvant radiotherapy: Analysis of efficacy and long-term toxicity. Haematologica 85:1032-1039, 2000

31. Santoro A, Bonadonna G, Valagussa $P$, et al: Long-term results of combined chemotherapyradiotherapy approach in Hodgkin's disease: Superiority of ABVD plus radiotherapy versus MOPP plus radiotherapy. J Clin Oncol 5:27-37, 1987

\section{Affiliations}

Marleen A.E. van der Kaaij, Arnold H.M. Simons, and Hanneke C. Kluin-Nelemans, University Medical Center Groningen, University of Groningen, Groningen; Pieternella J. Lugtenburg and Jeanette K. Doorduijn, Erasmus University Medical Center, Rotterdam; Berthe M.P. Aleman, the Netherlands Cancer Institute, Amsterdam; Evert M. Noordijk, Leiden University Medical Center, Leiden; Francisca Ong, Medisch Spectrum Twente, Enschede; Judith M. Roesink, University Medical Center Utrecht, Utrecht; John M.M. Raemaekers, Radboud University Nijmegen Medical Center, Nijmegen, the Netherlands; Natacha Heutte and Edwige Abeilard-Lemoisson, University of Caen-Basse Normandie; Christophe Fruchart and Michel Henry-Amar, Centre François Baclesse, Caen; Christophe Fermé, Institut de Cancerologie Gustave Roussy, Villejuif; Aspasia Stamatoullas, Centre Henri Becquerel, Rouen; Pauline Brice, Hôpital Saint Louis, Paris; Isabelle Gaillard, Hôpital Henri Mondor, Créteil; Serge Bologna, Centre Hospitalier Universitaire de Nancy Hôpital Brabois, Vandoeuvre-lès-Nancy; Houchingue Eghbali, Institut Bergonié, Bordeaux; Franck Morschhauser, Centre Hospitalier Régional Universitaire de Lille Hôpital Claude Huriez, Lille; Catherine Sebban, Centre Léon Bérard, Lyon; Marie Bouteloup, Centre Hospitalier Lyon Sud, Pierre Bénite, France; Paul Meijnders, Ziekenhuis Netwerk Antwerpen Middelheim, University of Antwerp, Antwerp; Anouk Allgeier and Bart Meulemans, European Organisation for Research and Treatment of Cancer, Brussels; José Thomas, Universitair Ziekenhuis Gasthuisberg, Leuven; Achiel Van Hoof, Algemeen Ziekenhuis Sint-Jan, Brugge, Belgium; Michele Spina, National Cancer Institute, Aviano, Italy; and Elizabeth C. Moser, Champalimaud Cancer Center, Lisbon, Portugal. 